\title{
EDUCAÇÃO PERMANENTE EM AGENTES COMUNITÁRIOS DE SAÚDE: EXPERIÊNCIA DE UM PROJETO DE INTERVENÇÃO
}

\author{
Bruno Bastos Godoi \\ Universidade Federal dos Vales do Jequitinhonha e Mucuri \\ bastosgodoi@gmail.com \\ Luciana Fernandes Amaro Leite \\ Universidade Federal dos Vales do Jequitinhonha e Mucuri \\ bastosgodoi@gmail.com
}

\section{Resumo}

O objetivo deste trabalho é relatar a experiência de discentes do curso de Medicina da Universidade Federal dos Vales do Jequitinhonha e Mucuri (UFVJM) em um projeto de intervenção que visou capacitar os Agentes Comunitários de Saúde (ACS) de uma Estratégia de Saúde da Família do município de Diamantina/MG. O projeto foi desenvolvido entre os meses de abril e julho de 2016 por oito discentes e uma docente do curso de Medicina da UFVJM. Foram realizados 10 encontros semanais com duração de 2 horas cada, cujo foco foi a capacitação dos ACS de temas escolhidos mediante prevalência de doenças da área de atuação da equipe de saúde. É de extrema importância a educação permanente em saúde dos ACS, quetem como objetivo influenciar positivamente nos indicadores de saúde da comunidade por meio de uma construção mais eficaz do conhecimento técnico-científico-reflexivo inerente ao dia a dia do profissional de saúde. Palavras-chave: Atenção Primária à Saúde. Capacitação. Educação Continuada. Estratégia de Saúde da Família.

\section{PERMANENT EDUCATION IN COMMUNITY HEALTH AGENTS: EXPERIENCE OF AN INTERVENTION PROJECT}

\begin{abstract}
The objective of this work is to report the experience of students of the medical course at the Federal University of Vales do Jequitinhonha and Mucuri (UFVJM) in an intervention project that aimed to train Community Health Agents (CHA) of a Family Health Strategy in the municipality of Diamantina/MG. The project was developed between the months of April and July 2016 by eight students and one professor from the UFVJM Medicine course. 10 weekly meetings were held with duration of 2 hours each, whose focus was the training of $\mathrm{CHAs}$ on themes chosen through the prevalence of diseases in the area in which the health team operates. It is of utmost importance the permanent health education of the CHA, which aims to positively influence the health indicators of the community through a more effective construction of the technicalscientific-reflective knowledge inherent in the daily routine of the health professional.

Keywords: Primary Health Care. Training. Continuing Education. Family Health Strategy.

\section{EDUCACIÓN PERMANENTE EN AGENTES COMUNITARIOS DE SALUD: EXPERIENCIA DE UN PROYECTO DE INTERVENCIÓN}

\section{Resumen}

El objetivo de este trabajo es informar la experiencia de los Estudiantes del curso de Medicina em la Universidad Federal de Vales do Jequitinhonha y Mucuri (UFVJM) en um proyecto de intervención dirigido a capacitar a Agentes de Salud Comunitaria (ASC) en una Estrategia de Salud Familiar em el municipio de Diamantina/MG. El proyecto fue desarrollado entre los meses de abril y julio de 2016 por ocho estudiantes y um profesor del curso de Medicina UFVJM. Se realizaron 10 reuniones semanales con una duración de 2 horas cada una, cuyo enfoque fuela capacitación de los ASC en temas elegidos a través de la prevalencia de enfermedad es en el área en la que opera el equipo de salud. Es de suma importância la educación permanente em salud de la ASC, cuyo objetivo es influir positivamente em los indicadores de salud de la comunidad a través de una construcción más efectiva del conocimiento técnico-científico-reflexivo inherente a la rutina diária del profesional de la salud.

Palabras clave: Atención Primaria de la Salud. Capacitación. Educación Continua. Estrategia de Salud de la Familia. 
Educação permanente em agentes comunitários de saúde: experiência de um projeto de intervenção

\section{INTRODUÇÃO}

As atribuições do Agente Comunitário de Saúde (ACS) podem ser sintetizadas nas atividades de identificação de situações de risco; orientação das famílias e comunidade e encaminhamento dos casos e situações de riscos identificados aos outros membros da equipe de saúde (HOLLANA, 2011; BRASIL, 2011, 2014, 2016; SOUSA; SANTANA, 2011; VIANA, 2018; VILLELA et al., 2017).

O trabalho do ACS auxilia no planejamento e na implementação das ações de saúde tanto localmente, ao encaminhar informações do território de abrangência para as Estratégias de Saúde da Família (ESF), quanto nacionalmente, alimentando dados dos sistemas de informação do Ministério da Saúde(ALONSO; BÉGUIN; DUARTE, 2018; BENDER et al., 2016; BRASIL, 2016; RODRIGUES; SANTOS; ASSIS, 2010).

Pesquisas que exploraram a percepção dos trabalhadores de linha de frente, como os ACS, expõem os problemas de inadequação do trabalho causados por projetos de sistemas de produção, processos, organização do trabalho e tarefas, feitos a partir de estereótipos simplificadores(GODOI et al., 2018; HOLLANA, 2011; MARZARI; SELLI, 2011; MENEZES et al., 2017; SOUSA; SANTANA, 2011; VIANA, 2018; VILLELA et al., 2017).

Os ACS possuem algumas atribuições que são de suma importância para o bom funcionamento da Atenção Primária em Saúde (APS): identificação de fatores socioeconômicos, culturais e ambientais que possam interferir na saúde da população de sua área; planejamento de ações estratégicas juntamente com a equipe; acompanhamento da comunidade por meio de visitas domiciliares; execução de atividades educativas individuais e coletivas e formação de grupos operativos(BENDER et al., 2016; GODOI et al., 2018; MARZARI; SELLI, 2011). Esses agentes conhecem profundamente a realidade local, acarretam vivências e experiências para o interior da equipe de saúde e abrem novos caminhos no processo de intervenção(ALONSO; BÉGUIN; DUARTE, 2018; SIMAS; PINTO, 2017; VIDAL; MOTTA; SIQUEIRA-BATISTA, 2015).

Ao definir os ACS como peça fundamental para o sistema de saúde, faz-se necessário evidenciar que eles precisam ser reconhecidos e valorizados pelo papel exercido na ESF,contudo, a capacitação destinada a esses atores é insuficiente e deficitária, não os preparando devidamente para atuar nos problemas com os quais se deparam durante o exercício de seu trabalho(ALONSO; BÉGUIN; DUARTE, 2018; APARECIDA CARDOSO, 2011; ROCHA et al., 2015; SANTOS; FILHO; FERNANDES, 2017). Diante do exposto, é notória a necessidade de investir cada vez mais na capacitação desses profissionais, a fim de proporcionar maior 
Educação permanente em agentes comunitários de saúde: experiência de um projeto de intervenção

eficiência nas funções de promoção da saúde e prevenção de agravos na comunidade adstrita pela ESF(DUARTE; DA SILVA; CARDOSO, 2007). O treinamento dos agentes deve muni-los de conhecimentos diversos e apropriados sobre o processo saúde/doença com a incorporação de saberes que os habilitem no processo de interação com as famílias e reconhecimento das necessidades. Dessa maneira, espera-se que os ACS sejam capazes de orientar a população corretamente sobre determinadas doenças e quando procurar auxílio médico(FROTA, 2017; GODOI et al., 2018).

Este artigo tem como objetivo relatar a experiência de discentes do curso de Medicina da Universidade Federal dos Vales do Jequitinhonha e Mucuri (UFVJM), em um projeto de intervenção educativa que visou capacitar os ACS de uma ESF do município de Diamantina/MG. Com isso, visa empoderar diretamente a população adscrita por meio das orientações dos ACS, após construção do conhecimento teórico, mediado pela reflexão crítica sobre determinadas mazelas que assolam a comunidade.

\section{MATERIAIS E MÉTODOS}

Trata-se de um relato de experiência de um projeto de intervenção educativa realizado entre os meses de abril e julho de 2016 em uma Estratégia de Saúde da Família do município de Diamantina/MG, seguindo características preconizadas para construção de um projeto de intervenção(HOLLANA, 2011). O projeto foi desenvolvido por oito discentes do curso de Medicina da UFVJM,coordenados e supervisionados por uma médica com formação na área de Medicina da Família e Comunidade. O projeto é vinculado às Práticas de Integração EnsinoServiço-Comunidade (PIESC), uma das unidades acadêmicas desenvolvidas pelos discentes do curso de Medicina ao longo da graduação.

O projeto foi desenvolvido em dez encontros semanais com duração de duas horas cada, cujo foco foi capacitar os ACS da ESF Saúde e Vida, situada na cidade de Diamantinano interior de Minas Gerais, nos temas escolhidos por eles mediante prevalência de doenças na área de atuação da equipe de saúde.

As temáticas escolhidas foram: "O papel do ACS no Contexto da ESF", "Doenças Transmitidas pelo Aedes aegypti”, "Calendário de Vacinação e Interpretação do Cartão de Vacina”, "Uso Racional de Medicamentos", "Orientações para Gestantes", “Obesidade", "O uso de drogas na adolescência" e "Estresse e Saúde Mental".

Participaram oito ACS, cuja média de idade era de 38 anos, com uma média de 8,5 anos de experiência e 4,6 anos como agente na atual ESF. Todos os agentes eram do sexo feminino e 
Educação permanente em agentes comunitários de saúde: experiência de um projeto de intervenção

possuíam pelo menos o ensino médio completo, sendo que três tinham superior completo e somente uma tinha ensino superior incompleto.

Para realizar a capacitação dos ACS utilizaram-semetodologias de ensino e aprendizagem ativas, como discussão dos temas por meio de brainstorming e relatos de conhecimentos prévios sobre o assunto (GODOI et al., 2018). Assim como de tecnologias de informação e comunicação (apresentação em power point), execução de dinâmicas de grupo,dramatizações, aplicação de jogos educativos e distribuição de materiais informativos.

As capacitações, com os respectivos horários, dias e temas a serem abordados, eram previamente pactuadas com o profissional de enfermagem responsável pela coordenação da ESF e também com os ACS, de maneira que estivesse presente o maior número de profissionais.

\section{RESULTADOS E ANÁLISES}

A escolha das temáticas do projeto "Capacitação de Agentes Comunitários de Saúde no município de Diamantina - MG" foi realizada em uma reunião com todos os profissionais da ESF para decidir quais seriam os temas que a equipe julgava pertinente. Decidiu-se que os temas a serem abordados ao longo das capacitações seriam: "O papel do ACS no Contexto da ESF", "Doenças Transmitidas pelo Aedes aegypti”, "Calendário de Vacinação e Interpretação do Cartão de Vacina”, “Uso Racional de Medicamentos”, “Orientações para Gestantes”, “Obesidade”, “O Uso de Drogas na Adolescência" e "Estresse e Saúde Mental". Para o embasamento teórico foram utilizados materiais preconizados pelo Ministério da Saúde (BRASIL, 2016).

As capacitações foram desenvolvidas com o uso preferencial de metodologias ativas que sejam aplicáveis ao tema trabalhado, por meio de problematização e discussão em mesa redonda, por meio da técnica de grupos operativos. Na semana que antecedeu cada capacitação, foi aplicado um questionário de múltipla escolha com temática relacionada ao assunto do próximo encontro. $\mathrm{Na}$ semana seguinte a cada capacitação, ao início do encontro, era aplicado o questionário referente ao tema da semana anterior. Ao fim de cada dia do projeto, era realizado feedback a fim de que houvesse um retorno imediato sobre as impressões coletivas do encontro.

Mediante avalição subjetiva dos discentes, observou-se uma melhora do envolvimento dos ACS e do conhecimento verificado a cada pós-teste. Isso proporcionou aproximação entre a equipe de saúde e os acadêmicos.

Além dos pontos positivos, identificaram-se pontos negativos e limitantes que retardaram a melhor execução do projeto: recusa de alguns ACS em relação aos discentes e baixa participação nas rodas de discussão. Supõe-se que esses pontos podem ter ocorrido devido ao 
Educação permanente em agentes comunitários de saúde: experiência de um projeto de intervenção

temor dos ACS em serem julgados pelo desconhecimento de temas rotineiros no ambiente da ESF. Essa situação foi superada mediante reuniões frequentes com toda a equipe de saúde, a fim de explicitar os objetivos da intervenção e da importância para a promoção em saúde e prevenção de doenças. O apoio da enfermeira chefe da ESF foi fundamental para sensibilizar os ACS da importância do projeto e da participação assídua nas capacitações.

Essa experiência proporcionou um aumento de vínculo entre a equipe de saúde e os estudantes de Medicina, o que possibilita os trabalhos desenvolvidos na ESF mais prazerosos e mais coesos, assim, capazes de identificar falhas e intervir eficazmente.

$\mathrm{Na}$ dinâmica do trabalho das equipes de saúde da família, o ACS é um profissional que merece destaque, cujas atribuições são funções estratégicas e complexas relacionadas à educação em saúde e divulgação de informações(GODOI et al., 2018). Para promover o desenvolvimento do processo de trabalho, é preciso criar estratégias de educação que encorajem a participação dos trabalhadores da área da saúde e assim possibilitem a capacitação profissional (CRUZ, 2017; FROTA, 2017; JÚNIOR, 2014; MARZARI; SELLI, 2011; VIDAL; MOTTA; SIQUEIRABATISTA, 2015). O processo de educação permanente dos ACS se faz presente na atual realidade brasileira. Frente ao avanço do Brasil em relação à da Atenção Primária e, consequentemente, da ESF, a educação do ACS, que está intimamente inserido na comunidade,deve evoluir de maneira semelhante, com vistas à qualificação da assistência prestada diretamente à população(GUERRA; MELO JÚNIOR; FROTA, 2018; MINISTÉRIO DA SAÚDE, 2011, 2014).

A educação permanente pode ser definida como um conjunto de atividades educativas para atualização do indivíduo, onde se oportuniza o desenvolvimento do trabalhador ea participação eficaz no dia a dia do serviço (GUERRA; MELO JÚNIOR; FROTA, 2018). Estabelecer um programa de educação permanente tendo como base a interdisciplinaridade propicia maior interação entre a equipe de saúde, permitindo a promoção da aprendizagem e o intercâmbio de conhecimentos(FROTA, 2017; MENEZES et al., 2017; MINISTÉRIO DA SAÚDE, 2016).

Várias são as possibilidades para a concretização da educação permanente, porém é importante ressaltar que o propósito de adquirir conhecimento, habilidades e mudanças comportamentais para o aprimoramento profissional e da assistência prestada devem fazer parte deste processo(ALONSO; BÉGUIN; DUARTE, 2018; BENDER et al., 2016; SIMAS; PINTO, 2017; SOUSA; SANTANA, 2011).

A Extensão Universitária é o processo educativo, cultural e científico que se articula com o Ensino e a Pesquisa de forma indissociável e viabiliza a relação transformadora entre 
Educação permanente em agentes comunitários de saúde: experiência de um projeto de intervenção

Universidade e Sociedade. Esse fluxo que estabelece a troca de saberes sistematizados, acadêmico e popular tem como consequências a produção do conhecimento, a democratização do conhecimento acadêmico e a participação efetiva da comunidade na atuação da Universidade(MARIA; MAIOR SERRANO, 2018).

Ao se fazer extensão, produz-se conhecimento que viabiliza a relação transformadora entre a Universidade e a sociedade e vice-versa (MARIA; MAIOR SERRANO, 2018).

A utilização de novas metodologias que proporcionaram a participação dos ACS durante as discussões contribuiu para a construção do conhecimento, sendo verificado pelos resultados obtidos nos testes pré e pós capacitação, visto a complexidade dos temas que rodeiam a prática diária dos ACS(GODOI et al., 2018; MARIA; MAIOR SERRANO, 2018).

\section{CONSIDERAÇÕES FINAIS}

O desenvolvimento do projeto "Capacitação de Agentes Comunitários de Saúde no município de Diamantina - MG” permitiu vivenciar a importância da comunicação que a extensão promove entre universidade e comunidade.

Foram enfrentadas dificuldades ao longo da execução do projeto, como a ausência em algumas capacitações ou baixa participação nas discussões, sendo possível superar barreiras, a fim de capacitar os ACS de maneira mais eficiente e completa.

Ao final do projeto foi possível notar o empoderamento dos ACS acerca dos assuntos desenvolvidos e de outras temáticas que surgiram ao longo das atividades desenvolvidas, o que proporcionou troca de experiências e construção do conhecimento entre a equipe de saúde da ESF e os discentes.

O desenvolvimento das capacitações possibilitou a obtenção de uma experiência única, na qual se pôde compreender nuances dos objetivos da ação de extensão universitária: promover uma transformação qualificada que acarrete em melhorias na qualidade de vida da comunidade onde a Universidade está inserida.

\section{REFERÊNCIAS}

ALONSO, C. M. do C.; BÉGUIN, P. D.; DUARTE, F. J. de C. M. Work of community health agents in the Family Health Strategy: Meta-synthesis. Revista de Saude Publica, v. 52, n. 14, p. 
Educação permanente em agentes comunitários de saúde: experiência de um projeto de intervenção

1-13, 2018. DOI 10.11606/S1518-8787.2018052000395.

APARECIDA CARDOSO, F. Capacitação de agentes comunitários de saúde: experiência de ensino e prática com alunos de Enfermagem. Revista Brasileira de Enfermagem, v. 64, n. 5, p. 968-73, 2011. Disponível em: http://www.scielo.br/pdf/reben/v64n5/a26v64n5. Acesso em: 28 dez. 2018.

BENDER, K. G. et al. Condições e modificações no processo de trabalho: concepções de Agentes Comunitários de Saúde. Revista Jovens Pesquisadores, v. 6, n. 2, p. 45-59, 2016. DOI 10.17058/rjp.v6i2.7283. Disponível em: https://online.unisc.br/seer/index.php/jovenspesquisadores/article/view/7283.

BRASIL. Ministério da Saúde. Diretrizes para Capacitação de Agentes Comunitários de Saúde em Linhas de Cuidado. 2016. Disponível em: www.saude.gov.br/bvs. Acesso em: 1 fev. 2020.

BRASIL. Ministério da Saúde. PORTARIA N 2.488, DE 21 DE OUTUBRO DE 2011. 2011. Disponível http://bvsms.saude.gov.br/bvs/saudelegis/gm/2014/prt0314_28_02_2014.html. Acesso em: 1 fev. 2020.

BRASIL. Ministério da Saúde. PORTARIA No 314, DE 28 DE FEVEREIRO DE 2014. 2014. Disponível em: http://bvsms.saude.gov.br/bvs/saudelegis/gm/2011/prt2488_21_10_2011.html. Acesso em: 1 fev. 2020.

CRUZ, G. D. M. DA. ESTRATÉGIAS DE CAPACITAÇÃO PARA AGENTES COMUNITÁRIOS DE SAÚDE NA FONOAUDIOLOGIA. 2017. Disponível em: https://repositorio.ufsc.br/xmlui/bitstream/handle/123456789/133446/Vanessa Martineli_TCC.pdf;sequence=1. Acesso em: 1 fev. 2020.

DUARTE, L. R.; DA SILVA, D. S. J. R.; CARDOSO, S. H. Construindo um programa de educação com agentes comunitários de saúde. Interface: Communication, Health, Education, v. 11, n. 23, p. 439-447, 2007. DOI 10.1590/S1414-32832007000300004.

FROTA, Y. B. da. Capacitação de agentes comunitários de saúde para orientação e acompanhamento dos usuários diabéticos e hipertensos cadastrados na equipe de saúde urbana III da UBS Gerson Kettle. 18 jun. 2017. Disponível em: https://ares.unasus.gov.br/acervo/handle/ARES/9174. Acesso em: 1 fev. 2020.

GODOI, B. B. et al. CAPACITAÇÃO DE AGENTES COMUNITÁRIOS DE SAÚDE NO MUNICÍPIO DE DIAMANTINA -MG. Revista Ciência em Extensão, v. 14, n. 1, p. 54-69, 2018.

GUERRA, H. S.; MELO JÚNIOR, C. A. D. C.; FROTA, R. S. Educação permanente para agentes comunitários de saúde: uma visão acadêmica. Extensio: Revista Eletrônica de Extensão, v. 15, n. 28, p. 101-107, 2018. DOI 10.5007/1807-0221.2018v15n28p101. Disponível em: https://periodicos.ufsc.br/index.php/extensio/article/view/1807-0221.2018v15n28p101.

HOLLANA, R. de P. G. Contrução de um projeto de intervenção em saúde bucal para uma escola municipal de cabo verde. 2011. Trabalho de Conclusão de Curso apresentado ao Curso de Especialização em Atenção Básica em Saúde da Família, Universidade Federal 
Educação permanente em agentes comunitários de saúde: experiência de um projeto de intervenção

de Minas Gerais, como requisito para obtenção do título de Especialista. Disponível em: https://www.nescon.medicina.ufmg.br/biblioteca/imagem/3019.pdf.

JÚNIOR, M. A. G. P. PLANO DE AÇÃO PARA CAPACITAÇÃO DAS AGENTES COMUNITÁRIAS DE SAÚDE DA UNIDADE BÁSICA DE SAÚDE SANTO ANTÔNIO EM MARIANA-MG. 2014.2 Disponível em: https://www.nescon.medicina.ufmg.br/biblioteca/registro/Plano_de_acao_para_capacitacao_da s_agentes_comunitarias_de_saude_da_Unidade_Basica_de_Saude_Santo_Antonio_em_Mariana MG/310. Acesso em: 1 fev. 2020.

MARIA, R.; MAIOR SERRANO, S. Conceitos de extensão universitária: um diálogo com Paulo Freire. 29 dez. 2018. Disponível em: https://issuu.com/praticasintegraisnutricao/docs/conceitos_de_extens_o_universit_r/14.

MARZARI, C. K.; SELLI, L. Agentes comunitários de saúde: perfil e formação. Ciência \& Saúde Coletiva, v. 16, n. 1, p. 873-880, 2011.

MENEZES, J. A. et al. Critical and Reflexive Training of Community Health Workers: a Strategy for the Active Professional Education in and for the Brazilian Unified Health System. DEMETRA: Alimentação, Nutrição \& Saúde, v. 12, n. 4, p. 849-864, 2017. DOI 10.12957/demetra.2017.28611.

ROCHA, G. S. S. et al. Conhecimento dos agentes comunitários de saúde sobre a tuberculose, suas medidas de controle e tratamento diretamente observado. Cadernos de Saude Publica, v. 31, n. 7, p. 1483-1496, 2015. DOI 10.1590/0102-311X00112414. Disponível em: http://www.scielosp.org/scielo.php?script=sci_arttext\&pid=S0102-311X2015000701483.

RODRIGUES, A. Á. A. de O.; SANTOS, A. M. dos; ASSIS, M. M. A. Agente comunitário de saúde: sujeito da prática em saúde bucal em Alagoinhas, Bahia. Ciência \& Saúde Coletiva, v. 15, n. 3, p. 907-915, 2010. DOI 10.1590/S1413-81232010000300034. Disponível em: http://www.scielo.br/scielo.php?script=sci_arttext\&pid=S1413-81232010000300034.

SANTOS, E. B.; FILHO, A. S. M.; FERNANDES, M. A. Análise do Conhecimento dos Agentes Comunitários de Saúde da Unidade de Saúde da Família de uma cidade do interior da Bahia sobre Hipertensão Arterial. Revista Multidisciplinar e de Psicologia, v. 11, n. 39, p. 432-451, 2017. .

SIMAS, P. R. P.; PINTO, I. C. de M. Trabalho em saúde: retrato dos agentes comunitários de saúde da região Nordeste do Brasil. Ciência \& Saúde Coletiva, v. 22, n. 6, p. 1865-1876, 2017. DOI 10.1590/1413-81232017226.01532017. Disponível em: http://www.scielo.br/scielo.php?script=sci_arttext\&pid=S1413-

$81232017002601865 \& \operatorname{lng}=$ pt\&tlng $=$ pt.

SOUSA, M. A. G. De; SANTANA, F. O Trabalho E a Qualificação Profissional Dos Agentes Comunitários De Saúde. EDUCERE, v. 1, n. 1, p. 10111-10123, 2011.

VIANA, A. D. O. Relato De Experiência De Ensino De Redação Para O Exame Nacional Do Ensino Médio (Enem) Em Um Projeto De Extensão De Cursinho Popular. Revista Brasileira De Extensão Universitária, v. 9, n. 2, p. 107-114, 2018. DOI 10.24317/23580399.2018v9i2.7778.

VIDAL, S. V.; MOTTA, L. C. de S.; SIQUEIRA-BATISTA, R. Agentes comunitários de saúde: Aspectos bioéticos e legais do trabalho vivo. Saude e Sociedade, v. 24, n. 1, p. 129-140, 2015. 
Educação permanente em agentes comunitários de saúde: experiência de um projeto de intervenção

DOI 10.1590/S0104-12902015000100010.

VILLELA, E. F. de M. et al. Educação em saúde : agentes comunitários de saúde e estudantes de medicina no controle da dengue. Revista Eletrônica de Comunicação, Informação e Inovação em Saúde, v. 11, n. 4, p. 1-8, 2017.

Recebido em: 21/08/2019

Aceito em: 30/03/2020 